\title{
Degradation Study of Archaeological Peruvian Fibers Through A Cleaning Process by SEM/Image Processing
}

\author{
Michelle Kearns*, Changmo Sung*, Irene Good** \\ *Department of Chemical Engineering-Center for Advanced Materials, University of Massachusetts, \\ Lowell, MA 01854 \\ **Peabody Museum of Archaeology and Ethnology, Harvard University, Cambridge, MA 02138
}

The use of image processing tools is growing in microscopy. The ease with which they allow microscopic images to be enhanced in terms of clarity and detail also allows for fast and accurate information abstraction from the image. Three archaeological fiber samples were examined with SEM/EDXS analysis, then cleaned and examined again. All the images were then processed with two image processing software programs. Measurements were made of fiber widths and the size of debris particles on the fibers using both programs. The results obtained from the programs were compared to each other, and the results for the different fiber samples were also compared to one another. The goal was to determine through visible analysis as well as quantitative measurements, which of the fiber samples was the most degraded and any changes that took place on the fibers after cleaning. The two software programs were also compared for their consistency and accuracy in measuring.

All fiber samples were sputter coated with gold and imaged with an AMRAY 1400 SEM at $5 \mathrm{kV}$ using a $\mathrm{LaB}_{6}$ filament. EDXS analysis was performed with a Noran analyzer at $20 \mathrm{kV}$. The fiber samples were cleaned by being brushed with a solution of seven drops of white vinegar in $100 \mathrm{ml}$ of deionized water. They were then brushed again with deionized water. Image analysis was performed with Scion image and GAIA Blue.

SEM analysis showed all fibers to be vegetable in origin, with two of the samples clearly identified as cotton. Figure 1 shows the difference in fiber surface appearance before and after cleaning. A tentative ranking of the samples according to degree of degradation was made, based on the visible integrity of the fibers as well as the intensity of debris coverage and penetration. Figures 2 and 3 show the fiber appearance after cleaning for the other two samples. The sample in Figure 2 exhibited measurable shrinkage of the fibers after cleaning. There were no discernable changes in the fiber width after cleaning for the other two samples, though the sample of Figure 1 had a lessening of large debris particles on the fibers. The elements common to all samples were $\mathrm{Si}, \mathrm{Ca}, \mathrm{Cl}$, and $\mathrm{K}$.

In general, EDXS showed no significant changes to the elemental analysis on the fibers after the cleaning, though the proportion of the elements to one another on a sample was affected. The heavy fibrillation of the sample as well as the thickness of its surface covering in Figure 3 shows it to be the most degraded of the three samples. Figure 1 shows the least degraded sample, determined by the flexibility of the fibers during cleaning, and the removal of much debris after cleaning. GAIA Blue provided more consistent results and therefore more accurate measurements for quantitative analysis of changes in fiber widths and debris size before and after cleaning.

\section{References:}

[1] D’Orazio, L. et al. Journal of Archaeological Science 27 (2000) 745.

[2] Peacock, E.E. International Biodeterioration \& Biodegradation (1996) 35. 


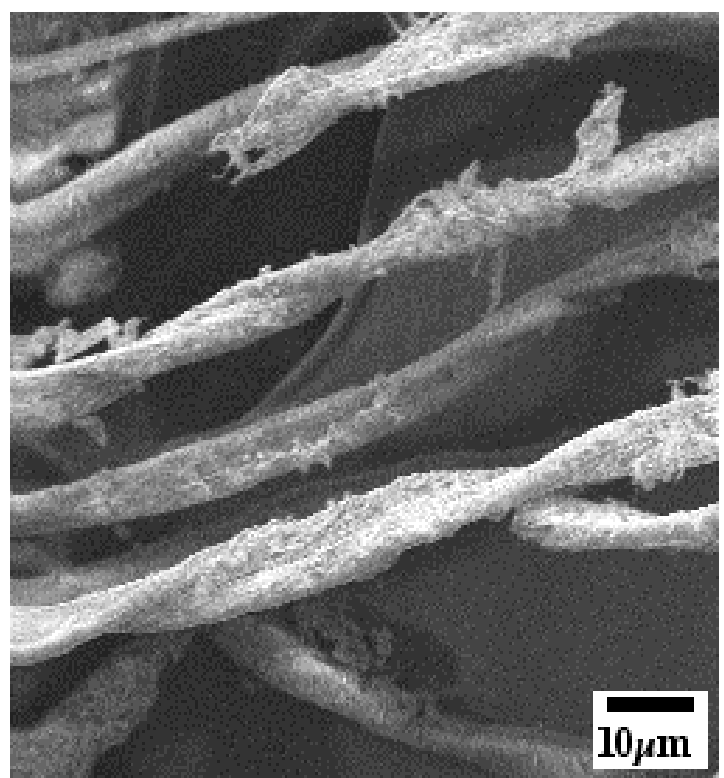

Fig.1a

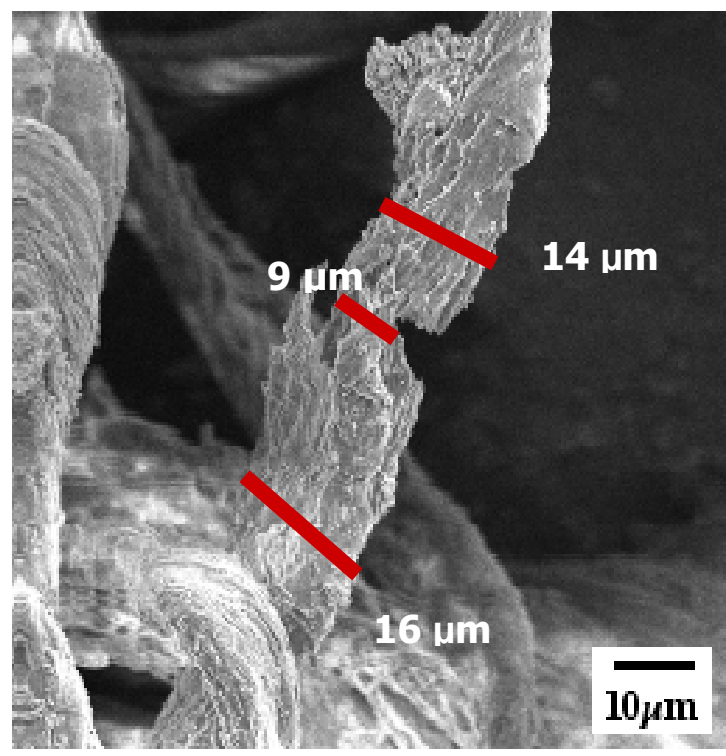

Fig.2

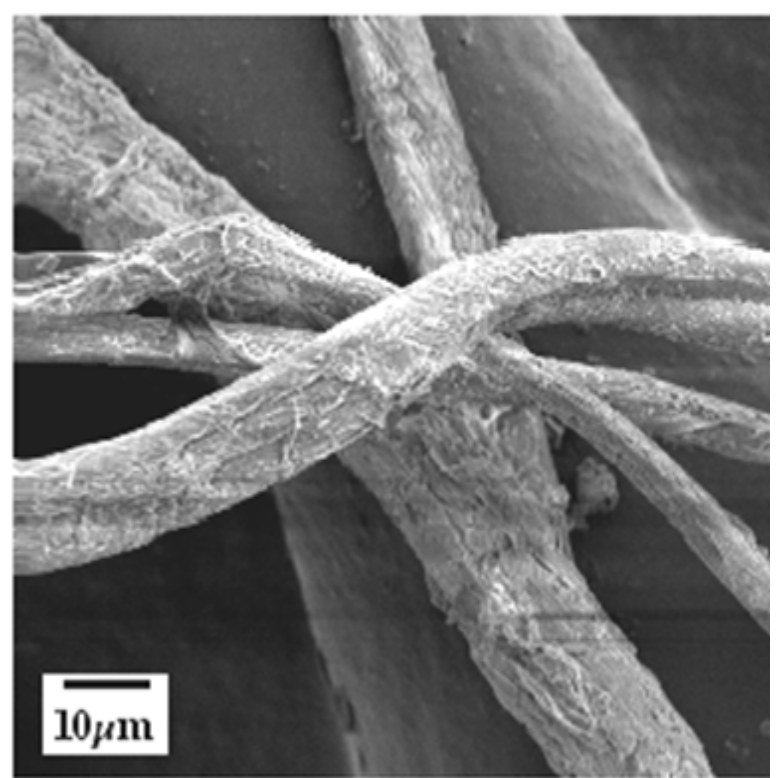

Fig.1b

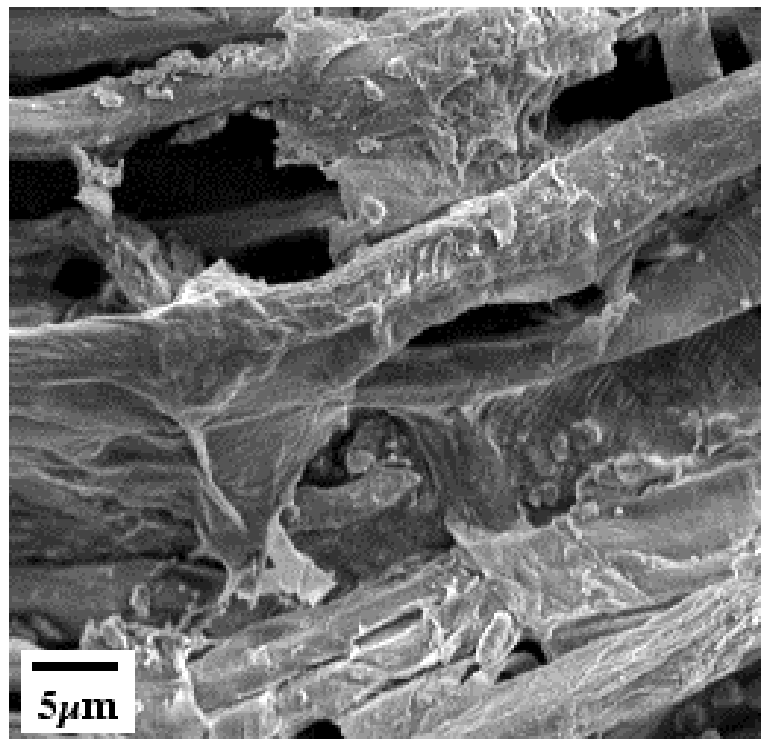

Fig.3

Fig.1. (a) SEM image of sample 976-42-30/11397-weft thread before cleaning. Sample identified as cotton fibers. (b) Same sample after cleaning. Fiber surface is more visible and appears intact, with a lessening of surface debris.

Fig.2. SEM image of sample 976-42-30/11404-weft thread after cleaning. Identified as cotton fiber. A coating on fibers is still visible, and fiber surface shows tears and holes. Image processing (GAIA Blue) identifies width of fiber at various points.

Fig.3. SEM image of sample 976-42-30/11416 after cleaning. Fiber is vegetable, but not cotton. Fiber surface is heavily obscured with coating, and fiber ends exhibit extensive fibrillation. 